IV Congreso Internacional Estética y Política Poéticas del desacuerdo para una democracia plural 16 y 17 de octubre. Valencia

Doi: http://dx.doi.org/10.4995/CEP4.2019.10537

\title{
Las voces del espacio público, una cuestión acerca de la democracia
}

\author{
Diana González Fonseca ${ }^{a}$
}

${ }^{a}$ Universidad Complutense de Madrid, dianamig@ucm.es

\begin{abstract}
In the emergency of thinking about public space and its relation to democracy, the following questions arise: how to overcome the limitations of individual identity and talk about a community? Is it necessary to evoke an unity in order to solve the ideal of equality? These are all difficulties inherent to the foundation of democracy and, consequently, to the possibilities that it enables in the public space. The consequences of one or another way of defining democracy affect the multivoiced body proposed by the American philosopher Fred Evans. This expression refers to the public as from heterogeneity, and introduces an aesthetic dimension to its political formula. Moreover, for the purpose of explaining the role of voices in the public space, I intend to mention democracy to come and the absolute hospitality by Jacques Derrida, on the one hand, and being singular plural by Jean-Luc Nancy, on the other. The democracy to come shows a perpetual tension between the similar and the other, which celebrates an ideal of democracy in eternal pursuit. On the other hand, being singular plural points at the necessity of the relationship with the other, of being-with which, saving the distance, reminds of Heidegger's Mitsein. These notions suggest a necessary openness of the public space and, ultimately, assign a simultaneous value to all voices.
\end{abstract}

Keywords: democracy, voices, hospitality, identity, public space

\begin{abstract}
Resumen
En la emergencia de pensar el espacio público y su relación con respecto a la democracia, surgen las siguientes cuestiones: ¿cómo superar las limitaciones de la identidad individual y hablar de una comunidad?, ¿es necesario invocar a la unidad para resolver el ideal de igualdad? Estas son dificultades inherentes a la fundamentación de la democracia y, por consiguiente, a las posibilidades que habilita en el espacio público. Las consecuencias de uno u otro modo de fundamentar la democracia afectan al cuerpo de multiples voces, propuesto por Fred Evans, filósofo estadounidense. Esta expresión sirve para aludir a lo público desde la heterogeneidad, además de introducir una dimensión estética a su fórmula política. Ahora, con el propósito de exponer el juego de las voces en el espacio público, propongo apelar a la democracia por venir y la absoluta hospitalidad de Jacques Derrida, por un lado, y el ser singular plural de Jean-Luc Nancy, por el otro. La democracia por venir muestra una tensión perpetua entre lo semejante y lo otro, en ella se celebra un ideal de democracia en persecución perpetua. En cambio, el ser singular plural indica la necesidad de la relación con el otro, de estar-con, que guardando las distancias, recuerda al Mitsein heideggeriano. Estas nociones sugieren una apertura necesaria del espacio público y, en último término, asignan un valor simultáneo a todas las voces.
\end{abstract}

Palabras clave: democracia, voces, hospitalidad, identidad, espacio público 


\section{Introducción}

La democracia y el espacio público son conceptos recíprocos. Las disposiciones de uno, repercuten en el otro invariablemente. En la emergencia de estudiar las condiciones de participación y representatividad en el espacio público, Fred Evans, filósofo estadounidense, propone la figura de las voces en representación de todos aquellos que participan de la ciudadanía. Las voces funcionan como una figura estética y política de los actores políticos de una sociedad democrática quienes conforman, en efecto, el cuerpo de múltiples voces. Esta teoría está influida por la filosofía contemporánea y, en particular, por el filósofo Jacques Derrida, quien ha influido la conformación general de la obra de Evans. La deconstrucción propuesta por Derrida es particularmente seductora porque advierte la insuficiencia de las instituciones y sugiere, no obstante, un proyecto de democracia: una democracia por venir. Este modo particular de entender la democracia aprehende las aporías y se apropia de sus paradojas; al tiempo que se autoconfirma, se autocritica. Al hacer converger la teoría de las voces con las premisas de la democracia por venir, Evans amplía la discusión hacia el terreno de lo espacial. Asimismo, Jean-Luc Nancy — quien no influye expresamente en la teoría de las voces - sugiere una manera ontológico-política de orientar las características intrínsecas de la comunidad. Nancy se apoya sobre la presunción de unidad y diferencia en la comunidad y discurre sobre las repercusiones que estas tienen en una política de lo "real" y lo situado de manera espacial.

El presente texto busca explorar los dilemas de fundamentación de la democracia, el papel de la democracia por venir y la posibilidad de asumir esta última en el espacio público. Para este propósito, me remitiré al libro más reciente de Evans, Public art and the fragility of democracy, y al libro Canallas: dos ensayos sobre la razón de Derrida. Asimismo, haré referencia a La comunidad inoperante y a Ser singular plural de Nancy en un intento de corresponder su ontología política con la figura del cuerpo de múltiples voces.

\section{Las voces en la democracia por venir}

El carácter aporético de la democracia supone, en palabras de Derrida (2005), hacer frente a una "fuerza sin fuerza, singularidad incalculable e igualdad calculable, conmensurabilidad e inconmensurabilidad, heteronomía y autonomía, soberanía indivisible y divisible o compatible, nombre vacío, mesianicidad desesperada o desesperante, etcétera". La democracia es a la vez el gran paradigma universalizable y un concepto equívoco sin referente; atrapada entre el ideal de igualdad y la salvaguarda de la diferencia — que a partir de ahora y en el resto del texto estará ligado al concepto de la differance derridiana - . Del mismo modo, la democracia por venir queda diferida del presente; sin embargo, está siempre pendiente y es siempre anticipada. De ahí que el filósofo contemporáneo francés encuentre un soporte en el sintagma "democracia por venir". A esta se le atribuye un estadio inacabado, es decir, que se adopta, pero a modo de proyecto. Desde la deconstrucción se plantea que "la expresión democracia por venir traduce sin duda o requiere una crítica militante y sin fin" (Derrida, 2005); es decir, que la democracia por venir no se logra, ni se alcanza; por el contrario, se aguarda. El estadio por venir no apunta a la imposibilidad de hacerse concreta como tampoco concede la función de una idea reguladora kantiana; sin embargo, está por venir "como la herencia de una promesa" (Derrida, 2005).

El porvenir de la democracia es el porvenir de lo otro, de la alteridad. A partir de este modo despersonalizado, se garantiza un ethos de hospitalidad que deja ver el manifiesto cosmopolita que defiende Derrida. Sin embargo, para explicar la hospitalidad es necesario hacer frente a una oposición: conceder hospitalidad ilimitada e incondicional en aquello que es incalculable — es decir, en las identidades culturales y sociales - no obstante, es preciso aplicar condiciones en aquello que es calculable —es decir, evaluar los riesgos para garantizar una regulación justa—. En esta condición que apela a la justicia, Derrida hace patente el vínculo que hay entre derecho y la democracia por venir. A este respecto dice: "Una política que no guarda una referencia con el principio de hospitalidad incondicional es una política que pierde su referencia con la justicia" (Derrida, 1994). Por ende, la justicia es un cimiento imprescindible del derecho.

Conforme a lo anterior, ¿es posible trasladar los preceptos de la democracia por venir a la noción de espacio público? De la hospitalidad incondicional se desprende una posible conjetura: que los espacios deben ser siempre receptivos, al tiempo que deben ser regulados para garantizar que las heterogeneidades permanezcan representadas. El espacio público involucra al ciudadano en la conformación de la opinión pública y le hace correspondiente de una voz. Esta voz no está mediada por una figura institucional ni por las categorías que una institución le pueda conferir, puesto que de hacerlo 
estaría comprometiendo su apertura política. Si las instituciones fallan es porque censuran, "excluyen a las personas y las reducen al silencio" (Derrida, 1994). En este sentido, bajo el precepto de la democracia por venir, el espacio público es un espacio de absoluta hospitalidad.

Evans (2018) introduce el concepto de voces para dar continuidad a su compromiso político y estético. Mediante este concepto el autor esquiva, por un lado, el dilema de la diversidad — en tanto rehúye la paradoja de la unidad frente a la diversidad - ; por otro lado, evade las tendencias autocráticas — visto que en lugar de reconocer formas instituidas, las trasgrede-. Estas dos dificultades están relacionadas con la deconstrucción de la figura del quién legitimado desde un régimen institucional. Estas figuras han sido asimiladas desde las instituciones y aparecen en categorías como el sexo, la raza o la religión. Asimismo, apelar a las voces significa reintegrar la audición en el espectro sensible y poner en pausa la posición jerárquica de lo visual. Tal y como explica Nancy el planteamiento estético, en cambio, habilita la noespecificidad de la libertad, en la que se ahondará más adelante.

Afirmar la dimensión estética de la sociedad repercute en los modos de fundamentar la posibilidad de la democracia. Evans advirtió la necesidad de hacer frente a las cuestiones de ciudadanía y el espacio público desde la política estética en aras de mantener un criterio amplio sobre las definiciones que de ella derivan. Lejos de intentar endurecerlas o declararlas terminadas, las definiciones que parten de lo estético presuponen la apertura hacia lo político. Para Evans (2018) las voces son las unidades primarias de la sociedad. Estas implican una conjugación estética y política desde lo corporal y, por lo tanto, la estructura social que construye en conjunto también es un cuerpo. Las voces son discursivas, intersubjetivas e interpelables. Evans (2018) explica:

El significado completo de la democracia y su espacio agnóstico permanece abierto debido al impulso que prima el diálogo, y a su mandato implícito de responder a otras voces y sus opiniones sobre la democracia. En otras palabras, la idea de este cuerpo democrático, de su carácter diacrítico (espacial) y agnóstico o dialógico (temporal), actúa como un aliciente constante para fomentar más lejos el debate sobre él y su significado, así como sus políticas gubernamentales. (trad. propia)

Si el autor propone las voces no es sólo para identificar a los participantes que poseen voz, sino para incluir en su espectro toda entidad capaz de fomentar el diálogo. De manera que una pieza de arte público es una quasi-voz, debido a que funciona como expresión o gesto enunciativo en el sentido del enunciado foucaultiano, es decir, como unidad operativa de sentido (Foucault, 2010). Las quasi-voces se implican en el diálogo político y expresan la opinión pública. De este modo, el espectro de las voces admite todo tipo de formas sensibles. Lo dialógico rebasa lo dicho y lo escrito como únicas formas legitimas de expresión política. En esta concepción se integran los textos del espacio público y todas sus formas. Evans subraya la tarea de preservar la apertura dialógica del espacio.

Ahora, ¿cómo asumir que la democracia por venir asiente un lugar en el espacio público para las voces? La respuesta está dada en la proposición de absoluta hospitalidad de la democracia por venir que, trasladada al espacio público, atiende las necesidades de proximidad, espaciamiento y acogida. Si tomamos en cuenta que la democracia por venir avista una comunidad de iguales compuesta de diferencia, A pesar de que Derrida no examina de cerca el espacio público, no es raro encontrar en su obra argumentos que se dirigen hacia aseveraciones sobre el espacio. Específicamente, la condición de hospitalidad incondicional implica dar pie a las heterogeneidades y asegurar completa apertura a las que están por venir, las que están por acontecer en el espacio. Derrida (2005) explica:

Esto implica otro pensamiento del acontecimiento (único, imprevisible, sin horizonte, que ninguna ipseidad, ni ninguna performatividad convencional y, por ende, consensual puede dominar), el cual se marca en un «por venir» que, más allá del futuro (puesto que la exigencia democrática no espera), nombra la venida de aquello que llega y aquel que llega, a saber, del arribante cuya irrupción en las fronteras de un Estado-nación civilizado no debería ni podría limitar ninguna hospitalidad condicional.

La cualidad del arribante como aquel que llega o que se instala, puede consentir, en consecuencia, una disposición de lo espacial en tanto receptáculo. Lo otro es el acontecimiento y el acontecimiento se materializa, se sitúa y se articula de forma espacial. La llegada del arribante implica necesariamente la transformación del espacio público debido a que su llegada supone una irrupción en una comunidad, un país, una familia o una doctrina, que traería consigo una ampliación 
de la opinión pública sobre la pertenencia a los espacios y sobre la cabida de los cuerpos que ahí se encuentran. El espacio tendría que asumir — bajo la apelación de la tolerancia incondicional — la opinión y la decisión del otro. Además, la imagen de la apertura — de aquello que se abre — se puede apreciar como una cualidad espacial en la que se establecen nuevas proximidades interpersonales, con un nuevo prójimo o un igual otro. Por último, cabe apreciar que los soportes de la democracia por venir brindan una enfoque esclarecedor sobre la inmigración, el control de fronteras y la condición política del extranjero en el mundo actual. Debido a una fuerte depuración de insignias institucionales, esta teoría vela por las virtudes políticas de tolerancia, respeto mutuo y solidaridad.

\section{Una comunidad de voces desde la ontología política}

La apuesta por una comunidad de voces que encuentran representación en el espacio público supone una condición trascendental. Si bien en el análisis anterior acerca de la democracia por venir se trazaron líneas remarcables en torno a la concepción de la comunidad, es a partir de Nancy que se atienden los pormenores desde una ontología política. Esto es posible a partir de la lectura que el filósofo hace de Heidegger en la que indaga, también, las implicaciones de una estética. A pesar de que esta ontología no hace una referencia expresa a la democracia, abarca rasgos que dan continuidad a lo tratado hasta ahora. La teoría de Nancy radicaliza la idea del Mitsein o ser-con heideggeriano con el fin de manifestar los rasgos de una comunidad anterior a toda subjetividad. Toda existencia es coexistencia — puesto que la comunidad es una condición originaria- y lo común es la evidencia del ser (Nancy, 2000). En sentido político, la ontología de Nancy acarrea consigo un modo de habitar.

El ser-con alude a la condición del ser que lo determina a ser uno-con-otro. Para Nancy la comunidad no es una entidad concertada bajo el reconocimiento de una identidad, es decir, bajo una semejanza de hecho; por ello, partir de lo común para entender la comunidad no sería adecuado en este análisis. Por el contrario, la idea de lo común precede a lo singular. En palabras de Nancy (2006): "Lo singular es un plural". No hay un acto de unión que conforme la comunidad, como tampoco hay un acto de separación de lo colectivo del que se desprenda el individuo. En cambio, la idea de comunidad se fundamenta desde una condición ontológico-estética. No hay un paso para transitar de lo individual hacia lo social, sino que la comunidad es necesaria en toda unidad del ser, es decir, que la comunidad es operativa en la existencia, en palabras de Nancy (2000):

[...] no hay comunión, no hay ser común, hay el ser en común. Toda la ontología, desde el momento en que es esta lógica del ser-en-sí como ser a sí, se reduce de este modo al en-común del a-sí. [...] El sentido del ser no es común — sino que el en-común del ser transita todo el sentido.

Para Nancy la libertad del ser consiste en la partición de sí mismo en singularidades; de esta manera, son los aspectos singulares los que determinaran la cosa. La libertad es la condición que posibilita que el ser se inmiscuya en el juego de las relaciones, juego que abre el mundo, retira el ser y deja vía libre para la expresión de la cosa y su finitud radical así como constitutiva. Nancy encuentra un vínculo entre existencia y relación, entre existente y sustancial, ya que en el tránsito del ser a lo ente hay lo que Nancy llama la partición o partager. Al hecho de la existencia precede una diferenciación, esta es, la partición o el acontecer; es la venida de la relación que hace posible el espaciamiento y la cristalización de las subjetividades en la libre asociación de lugares.

El espaciamiento es, también, un desajuste necesario en el espacio del sentido — entendido como sentido del mundo- y en la disposición de las cosas. La partición ontológica otorga la necesidad de espaciamiento en la presencia; en otras palabras, en la partición del ser, la presencia separa, brinda un espaciamiento que da cabida a lo singular. Asimismo, lo singular adquiere la condición de estar-juntos de la presencia. Nancy (2000) explica que "La comparecencia de los seres singulares —o de la singularidad del ser — mantiene una distancia abierta, un espaciamiento, en la inmanencia". Entonces, el espaciamiento está relacionado con llegar-a-ser y el devenir de lo singular, que mantiene una relación de inmanencia por el ser-para-la-muerte — concepto retomando de Heidegger- La partición es el reparto de los límites, de la entrega al exterior o del abandono que, al cabo, entrega lo propio, lo personal, lo social, lo público y lo corpóreo. Por ende, no es posible hablar de espacio público sin asumir esta partición. 
Ahora, en la condición de habitar en el espacio, los vínculos — a veces rotos- de los seres singulares son comunicativos, íntimos y orgánicos. Los seres singulares adoptan la forma comunicativa inteligible en el desarrollo de las identidades políticas. En la facultad de comunicarse, las voces — como unidad - se hacen distinguir en la diferencia; esta constituye el diálogo, la oposición y las bifurcaciones que, luego, constituyen también un cara de lo público. Nancy liga la diferencia con el acontecimiento, de una forma que no dista demasiado de las consideraciones derridianas tratadas en el apartado anterior. El filósofo francés explica que la articulación de lo que ha sido diferenciado se especifica en el cuerpo y en lo sensible, características presentes en lo "real". El cuerpo no funge como materia sino como figura politizada de la finitud. El cuerpo es la expresión de la diferencia, luego, el cuerpo es la voz. Por ello, una comunidad no es más que el lugar de todas las voces fragmentado en cada cuerpo, en cada subjetividad.

Para Evans, el régimen estético en el que se inserta el cuerpo de múltiples voces involucra la subjetividad y la interacción libre de las voces — pensados como modos discursivos-. Esta interacción supone la apertura del espacio y su derivación en espacio público. Los miembros del cuerpo social encuentran en la diferencia un campo de combate en el que se disputan las identidades políticas de los sujetos. Estas contiendas se sitúan en el espacio público, desde el movimiento y la defensa -o ataque - hacia el cuerpo otro. Evans se aproxima a la teoría de Nancy cuando indica que la caracterización del cuerpo social sucede en el acontecimiento, por lo tanto, el cuerpo siempre está en conflicto consigo mismo (Evans, 2018). La alteridad puede acontecer en el cuerpo de hecho — en el presente - debido a que una voz no se limita a ser un discurso fijo e invariable; por el contrario, se define como emergente e incondicional, incluso espontáneo. La disposición de un espacio público abierto conserva los preceptos de la partición tal y como ha sido descrita por Nancy.

\section{Conclusiones}

La teoría de Evans - moldeada alrededor de la figura de la voz y el cuerpo de múltiples voces— consigue una posición afirmativa sobre la democracia y sobre la posibilidad de conducir el proyecto democrático hacia los márgenes del espacio público. La comunión entre ésta y la democracia por venir de Derrida se hace manifiesta en la proyección de la hospitalidad en el espacio público; primero, porque sostiene la apertura espacial permanente e ilimitada y; segundo, porque establece la incondicionalidad de pertenencia en los asuntos públicos a quien se haga presente cualquiera que sea su posición en la opinión pública. De este modo, se asegura la representación de todas las identididades y, explícitamente, de aquellas que están por venir. La correspondencia entre la voz — como unidad social— y el espacio público — como receptáculo de expresiones políticas - cobra relevancia cuando se apela a lo dialógico de los enunciados y a la capacidad de la voz para presentarse en más de una forma sensible. El juego dialógico y discursivo genera proximidades al tiempo que resgurada los espaciamientos propios de la diferencia.

En adición, el recorrido que une a Derrida con Nancy yace en la configuración de la comunidad, por una parte, en términos políticos y, por otra parte, bajo supuestos ontológico-políticos. Ambos priman una fundamentación anterior al hecho que no establece parámetros de actuación ni formas establecidas institucionalmente. En cambio, describen propiedades intrínsecas a lo político. La postura de ambos con respecto a la alteridad es equiparable a la noción de acontecimiento. Para Derrida, lo otro es el acontecimiento que posibilita la democracia por venir, del mismo modo que para Nancy acontecer en la diferenciación es la evidencia misma de lo común. En este sentido, la conformación de identidades políticas que se disputan en el espacio público es entendida como la máxima expresión de la partición. Las voces han sido dispuestas como cuerpos en posesión de cualidades sensibles que les permiten distinguirse en la diferencia y, finalmente, están confinadas a habitar en comunidad.

\section{Referencias}

DERRIDA, J (2005). Canallas. Dos ensayos sobre la razón. Madrid: Editorial Trotta.

DerridA, J. “Deconstruir la actualidad” en El Ojo Mocho. Revista de Crítica Cultural. Buenos Aires (1994, 5, 60-75).

DERRIDA, J. y Fernández, E. (1994). “A democracia é uma promessa” en Jornal de Letras, Artes e Ideias. Lisboa (1994, 9-10).

Evans, F. (2018). Public art and the fragility of democracy: an essay in political aesthetics. Nueva York: Comlumbia University Press.

Foucault, M. (2010). La arqueología del saber. México: Siglo XXI. 
NANCY, J-L. (2000). La comunidad inoperante. Snatiago de Chile: Libros ARCES-LOM.

NANCY, J-L. (2006). Ser singular plural. Madrid: Arena Libros. 